\title{
Frequency and latency of response to offset-onset sequences of cutaneous stimulation
}

THOMAS G. STICHT, UNIVERSITY OF LOUISVILLE THOMAS E. SITTERLEY 1 , UNIVERSITY OF ARIZONA

\begin{abstract}
Beginning with a mechanically deforming stimulus resting on the skin, the duration of the temporal interval between the offset of the stimulus and the subsequent onset was varied from 45 to $140 \mathrm{msec}$. Response frequency and latency measurements were obtained for well trained Ss for each offset-onset sequence of stimulation. The results indicated that, (1) response frequency drops to below threshold with durations below 75 msec., and (2) RT increases as the duration is decreased. The results are discussed in terms of the recovery movement of the skin following the removal of the stimulus.
\end{abstract}

\section{Introduetion}

Several investigators (Nafe \& Wagoner, 1941; Kirk \& Kvorning, 1949; Kirk \& Chieffi, 1962) have demonstrated that considerable time may be required for the skin to recover its original contour following the offset of a mechanically deforming stimulus. Thus, if the skin is repeatedly stimulated, as in vibrotactile stimulation, it is likely that the skin would not be able to recover much of its contour between successive offsets and onsets of the stimulus. Geldard (1962, p. 15) has referred to this problem in terms of the "following" characteristics of the skin. Summarizing work conducted over a 14 year period, Geldard states that, "No investigation ... was able to show any effect on sensory processes of failure of the skin to follow the contactor motion." He then goes on to point out that the lack of showing such effects does not mean that such effects do not exist, but that they have not been demonstrated with the psychophysical techniques used.

In the present study, discrete, unitary stimulation is used and the "following" characteristics of the skin are investigated in relation to frequency and latency of response to such stimuli.

\section{Method}

In this study, a tactual stimulator (cf., Sticht, 1964) consisting of a stainless-steel needle of $0.5 \mathrm{~mm}$ diameter was adjusted, with the aid of a micrometer, above the palmer aspect of S's right middle finger. When the solenoid to which the stimulus was attached was activated, the stimulus was plunged $0.02 \mathrm{~mm}$ into S's finger. S's finger was inserted into a plastic cube mounted on an arm rest. This cube had a $3 \mathrm{~mm}$ hole in its midline. When $\mathrm{S}$ inserted his finger into the cube, a small area of skin protruded through the hole and remained there in a stable manner during the rather abrupt movements of S's right arm, which tended to occur whenever $\mathrm{S}$ reacted with a gross movement of his left arm.
The sequence of stimulation was as follows: first the stimulus was placed $0.02 \mathrm{~mm}$ into S's finger and allowed to remain there, statically, until adaptation occured. The stimulus was then rapidly removed, left off of the finger for some variable duration, and was then rapidly replaced. The duration of the temporal interval between the offset and the subsequent onset of the stimulus was varied as 45, 55, 75, 105 and $140 \mathrm{msec}$. (this temporal interval will be referred to as the off duration). In addition to the foregoing off durations, a 65 msec. off duration was used with $\mathrm{S}$ T.S. in an ad hoc manner after inspection of his data in order to confirm the shape of the obtained relations. Calibration of the off durations was accomplished from measurements of oscilloscopic tracings of the stimulus movement, as picked-up by a photocell. An error range of $\pm 5 \mathrm{msec}$. in the off durations was indicated.

Each stimulus presentation was preceded by a variable, random foreperiod ranging from 2.0 to 3.5 sec. Stimulus foreperiods and durations were controlled by two Hunter decade interval timers, models 111-C and 100-C respectively. Reaction times were recorded with a Hewlett-Packard model 522B electronic counter. During the experimental sessions, Ss (two male graduate students, well practiced in reacting to cutaneous onset and offset stimuli) were seated in a light-secure, ventilated booth. An armrest in the booth held S's reaction key. Ss responded whenever they felt any activity on their finger. This they did by releasing the micro-switch lever, causing the electronic counter, which started counting time at the offset of the stimulus, to stop counting. Reaction times were then recorded from the counter. A total of 50 trials per off duration was presented. Each trial was observed with the aid of a $25 x$ binocular microscope, which was also used in positioning the stimulus on S's finger.

\section{Results and Diseussion}

The microscopic observation of each stimulation sequence revealed that $\mathrm{Ss}$ failed to react to the offset of the stimulus, and instead responded to the subsequent onset. In other words, the offset was subliminal. This meant that the duration of the off period was added to each S's RT. When the off duration is subtracted from each S's mean RT, the resulting figure is the RT to the onset of a stimulus which has been preceded at varying intervals by a prior deformation of the skin. Stated differently, this "corrected" RT represents the effects of limiting the extent of "rebound" of the skin before it is deformed again. The mean "corrected" 
RTs for the 75,105 , and $140 \mathrm{msec}$. off durations were 271, 261, and $253 \mathrm{msec}$. for S T.S. and 311, 328, and 298 msec. for G.S. Except for the deviant score obtained with the $105 \mathrm{msec}$. off duration, the "corrected" RTs indicate that RT increases as the interval between the offset and subsequent onset is decreased.

None of the off durations were accompanied by $100 \%$ responding. Ss responded about $90 \%$ of the time with the three longest off durations. Response frequency dropped to below threshold (i.e., 50\% response) with off durations below $75 \mathrm{msec}$. Since response frequency was below threshold with the briefer off durations, it was not possible to compute even median RTs for these conditions. Therefore, in order to indicate the effects of decreasing the off duration on tactual sensitivity, frequency of response curves were prepared for each $S$ (Fig. 1). It is apparent from Fig. 1 that responsivity decreases abruptly with durations below $75 \mathrm{msec}$. Responses to the 65 msec. off duration with S T.S. were obtained at the end of the experiment and support the shape of the curves. As indicated, response frequency is highly similar for the 75, 105 and $140 \mathrm{msec}$. off durations. This suggests a similar level of sensitivity to onset stimulation following these durations, and is perhaps reflected in the slight differences in the "corrected" RTs obtained with these off durations. Because of the tenuous nature of the RT data, it is best to regard them as collateral support for the frequency of response data.

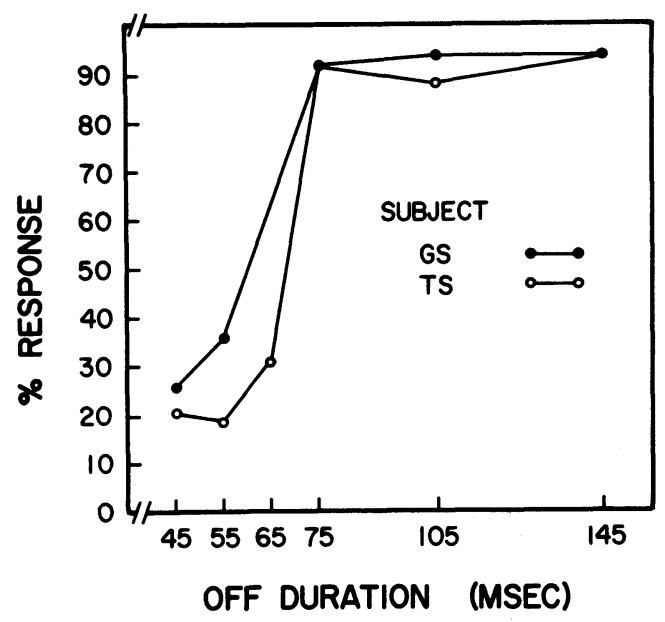

Fig. 1. Percent response to fifty stimulus presentations using the off durations shown on the abscissa.
These findings are consistent with the theory suggested by Kenshale \& Nafe (1962), which states that the adequate stimulus for touch is movement of the skin tissues above some minimal rate. It follows from the movement theory that the greater the amount of tissue movement, the stronger the consequent stimulation and the more intense the touch sensation. Nafe \& Wagoner (1941b) have included rate of movement, volume of tissue moved and the extent to which it is moved (i.e., the amplitude of deformation) in the expression "amount" of movement. In the present study, decreasing the off duration appears to have had the effect of decreasing the amount of tissue available to be pushed down at a supraliminal rate with the subsequent onset of the stimulus. The effect is the same as decreasing the amplitude of deformation, i.e., RTs increase (cf., Sticht, 1964) and thresholds (i.e., frequency of response) increase (c.f., Guilford \& Lovewell, 1936).

The present results also indicate that the "following" characteristics of the skin may, indeed, attenuate the touch sensation. Prior failure to establish this finding (Geldard, 1962, p.15) may be due to the almost exclusive use of repeated, vibrotactile stimulation. Such stimulation introduces complicated physiological processes of summation, inhibition, fatigue, etc. which perhaps conceal any contribution of the recovery movements of the skin. Using unitary stimulation, it remains to be demonstrated how the variation of such parameters as amplitude of deformation, area of stimulation, and off durations briefer than those used herein affect the response to offset-onset sequences of stimulation.

Referenees

Geldard, F. A. Virginia cutaneous project. 1948-1962. ONR Report NR-140-598, 1962.

Guilford, J. P., \& Lovewell, E. M. The touch spots and the intensity of the stimulus. J. gen. Psychol., 1936, 15, 149-159.

Kenshalo, D. R., \& Nafe, J. P. A quantitative theory of feeling. Psychol. Rev., 1962, 69, 17-33.

Kirk, J. E., \& Chieffi, M. Variation with age in elasticity of skin and subcutaneous tissue in human individuals. J. Gerontol., $1962,17,373-380$.

Kirk, J. E., \& Kvorning, S. A. Quantitative measurements of the elastic properties of the skin and subcutaneous tissue in young and old individuals. J. Gerontol., 1949, 4, 273-284.

Nafe, J. P., \& Wagoner, K. S. The nature of pressure adaptation. J. gen. Psychol., 1941, 25, 323-352.

Nafe, J. P., \& Wagoner, $\mathbf{K}$. S. The nature of sensory adaptation. J. gen. Psychol., 1941b, 25, 295-322.

Sticht, T.G. Reaction time to cutaneous onset and offset stimulation. Percept. mot. Skills, 1964, 19, 611-614.

Note

1. This research was supported in part by National Science Foundation grant GB 1384 to the University of Arizona under the direction of Dr. Neil R. Bartlett. 